\title{
Non-tuberculous micobacteria and leprosy
}

\section{Buruli ulcer, a reemerging mycobacterial disease}

\author{
Françoise Portaels
}

Mycobacteriology Unit, Department of Microbiology, Institute of Tropical Medicine, Nationalestraat 155 B-2000 Antwerpen, Belgium.

Buruli ulcer (BU), caused by Mycobacterium ulcerans, is an indolent necrotizing disease of the skin, subcutaneous tissue and bone. BU is the third most common mycobacterial disease of humans, after tuberculosis and leprosy, and the least understood of the three diseases.

$\mathrm{BU}$ is endemic in Africa, particularly in West African countries. The disease is also endemic outside Africa, but remains uncommon in non-African countries. Several imported and exported cases have also been described.

The epidemiology of BU is strongly associated with wetlands, especially those with slow-flowing or stagnant water (ponds, backwaters and swamps).

Recently, aquatic insects have been considered as potential passive reservoirs or 'vectors' of $M$. ulcerans. For the first time, a fully characterized M. ulcerans strain has been cultivated from an aquatic Hemiptera (Gerris) supporting the concept that the agent of $\mathrm{BU}$ is a human pathogen with an environmental niche.

The use of protected sources of water for domestic purposes reduces exposure to $M$. ulcerans contaminated sources and consequently may reduce prevalence rates of $\mathrm{BU}$.

Since BU was declared an emerging disease in 1998, much effort has been invested in research. Some aspects, however, remain unclear and thus require much more investigation, including reservoir(s) and mode(s) of transmission, risk factors, optimal management and preventive tools. Better strategies for early diagnosis and effective therapy compatible with the socioeconomic structures of BU endemic areas, should be developed. A multidisciplinary approach and productive cooperation between scientists and health professionals remain indispensable for the improvement of $\mathrm{BU}$ control worldwide.

\section{Mycobacterium terrae complex re-examined: old phenotypic knowledge and modern genotypic findings face to face}

\author{
Enrico Tortoli
}

Regional Reference Center for Mycobacteria, Florence, Italy.

The Mycobacterium terrae complex is considered a minor group within the genus Mycobacterium. It was initially conceived as a container to park three species (Mycobacterium terrae, Mycobacterium nonchromogenicum and Mycobacterium triviale) which could not be distinguished with classical biochemical and cultural 
tests. Among such species, which are unpigmented and characterized by intermediate growth rate, mainly $M$. nonchromogenicum has been reported, although rarely, involved in human diseases. Genetic investigations, based on 16S rDNA, first demonstrated the unrelatedness of $M$. triviale with other species and, more recently, lead to the recognition of four novel species within the complex (Mycobacterium hiberniae, Mycobacterium arupense, Mycobacterium kumamotonense and Mycobacterium senuense). With the aim of improving present knowledge of M. terrae complex we investigated, both phenotypically and genotypically, about 90 independent strains isolated from clinical specimens in the last 15 years. Genotypic analysis included sequencing of $16 \mathrm{~S}$ rRNA, hsp65 and $r p o B$ genes, while phenotypic investigations mainly aimed to HPLC profile of cell wall mycolic acids and to major biochemical and cultural features. A striking picture, characterized by the contrast between the heterogeneity at genetic level and the homogeneity of phenotypic features emerges.

\title{
Soft tissue infection due to non-tuberculous mycobacteria: epidemiology, diagnosis and treatment
}

\author{
Jacobus H. de Waard
}

Laboratorio de Tuberculosis, Instituto de Biomedicina, Universidad Central de Venezuela, Caracas, Venezuela.

This seminar discusses the clinical and epidemiological characteristics, the microbiological diagnosis, the treatment and follow-up of patients from Venezuela with soft tissue infection caused by non-tuberculous mycobacteria. Most cases seen in our laboratory were caused by mesotherapy or following plastic surgery. Mycobacterium abscessus, $M$. chelonae and $M$. fortuitum were the most common species, but $M$. simiae and a new species that was designated "M. cosmeticum" were also isolated. In our laboratory mycobacteria are identified by PCR restriction fragment length polymorphism analysis of the hsp65 gene. We discuss the application of this method for the direct identification in clinical samples and present the first cases of $M$. genavense y $M$. haemophilum infection in Venezuela. Patients were treated with species-specific antibiotic agents for 3 to 18 months. Investigation into the source of the infection revealed that patients were clustered within different outbreaks and products were found to be contaminated with Mycobacteria. Furthermore, failures in disinfection procedures were responsible for outbreaks. Several disinfectants available in Venezuela, used in clinical settings and registered as tuberculocidal, were tested for their mycobactericidal activity and were found not effective against mycobacteria. We inform that the use of these products for disinfecting critical and semi-critical material could result in iatrogenic infections. We conclude that physicians should be alerted to the possibility of infection by non-tuberculous mycobacteria in patients with a history of mesotherapy or plastic surgery who develop late-onset skin and soft tissue infection, particularly if they do not respond to conventional antibiotic treatment. 


\title{
Why monitoring and controlling leprosy patients after poly chemotherapy
}

\author{
Michel Faizal \\ Department of Medicine, Universidad Nacional de Colombia-Medical School. Bogota, Colombia. \\ South America. \\ Patients who receive therapy, specially during the first two years after specific \\ treatment is initiated, are in risk of develop leprae-like reactions, as well as silent \\ neuropathy, relapse, resistance to PQT-MB, scamous cell carcinoma of the skin, \\ plantar wounds, chronic renal insufficiency, and progression of disability. Based in \\ these considerations, control is recommended every six months along five years \\ of follow-up in paucibacillary patients, and every six months along ten years of \\ follow-up in multibacillary patients.
}

Evaluation must include a full clinical record emphasizing the examination of ocular, otorynolaryngological, osteoarticular, genitourinary and peripheral nervous systems. It is also important to exclude leprotic reactions as well as signs of relapse and resistance to treatment.

The relatives and must be followed and must be evaluated periodically at least two times a year in order to exclude infection as asymptomatic or subclinical carriers or if they have developed overt disease.

Paucibacillary patients could be discharged from the program once the treatment is finished and the period of five years of follow-up is completed without clinical or microbiological evidence of relapse. The same recommendations are given for the multibacillary patients, except for the fact that the follow-up must be extended during ten years.

Leprosy patients have a very high risk of developing psychiatric disorders (6 to 84 per cent) mainly depression, somatomorphic alterations and anxiety disorders $(48,49)$. The treatment and rehabilitation of these patients must include then, periodic mental health status evaluations.

To address treatment and follow-up of this group of patients must be an interdisciplinary effort with special emphasis in patient, community and health personnel education, and specialized psychiatric treatment. 